\title{
El aura y las formas de pensamiento en la pintura abstracta de las Primeras Vanguardias
}

\author{
Iñigo Sarriugarte Gómez \\ Universidad del País Vasco
}

RESUMEN

La publicación de una serie de manuales teosóficos a finales del siglo XIX y principios del XX sobre cuestiones tan novedosas como la tipología de las formas del pensamiento y lo referente a la composición cromática del concepto del aura, supuso para numerosos creadores vanguardistas la apertura de nuevas posibilidades experimentales. En este sentido, algunas de las propuestas pictóricas de Wassily Kandinsky, Theo van Doesburg y František Kupka, entre otros, se podrían interpretar como permutaciones cromáticas a partir de la estipulación teórica que generaron especialmente los teósofos C.W. Leadbeater y Annie Besant. Sin realizar una traslación mimética de las diferentes conjeturas planteadas en las creencias de esta escuela de pensamiento hermético, dichos artistas se embarcaron en la proyección de nuevas oportunidades experimentales a la vez que se sustentaban en la validación de un corpus teórico que justificaba la práctica abstracta en relación con un entramado de mayor acercamiento espiritual.

\section{PALABRAS CLAVE}

Teosofía, forma de pensamiento, aura, abstracción, vanguardia.

The Aura and the Thought-Forms in the abstract painting of Avant-Garde.

ABSTRACT

The publication of some theosophical manuals in the late nineteenth century and early twentieth century on novel issues like the type of thought-forms and the color composition of concept aura, meant the opening new creative possibilities for many avant-garde artists. In this sense, some of the paintings by Wassily Kandinsky, Theo van Doesburg and František Kupka, among others, could be interpreted as chromatic permutations from the theoretical stipulation generated especially by the theosophists CW Leadbeater and Annie Besant. Without realizing a mimetic translation of the different hypotheses and conjectures based on the beliefs of this school of hermetic knowledge, these artists embarked on the projection of new experimental opportunities while they were based on the validation of a theoretical corpus that justified the abstract practice in relation to a framework of greater spiritual weight.

KEY WORDS

Theosophy, Thought Form, Aura, Abstraction, Avant-garde. 


\section{Introducción al aura y a las formas de pensamiento en la teosofía}

Tanto los planteamientos teóricos de H.P. Blavatsky en la Doctrina Secreta (1888) como las posteriores perspectivas hipotéticas de C.W. Leadbeater, Annie Besant y Rudolf Steiner aludían en numerosas ocasiones al concepto de aura y las formas del pensamiento. En este sentido, las principales aportaciones teóricas y visuales que tuvieron muchos de los vanguardistas de principios del siglo XX fueron las obras tituladas El hombre visible e invisible (1902) de C.W. Leadbeater; Formas del Pensamiento (1901) del anterior autor junto con Annie Besant; y finalmente el trabajo del antropósofo Rudolf Steiner ${ }^{1}$ bajo el epígrafe Teosofía: una introducción a los procesos espirituales en la vida humana y en el cosmos (1904).

Tanto el concepto de aura como el de las formas de pensamiento tienen una vinculación directa con la teoría septuble de la naturaleza del ser humano, que se maneja en los ámbitos teosóficos, donde en base a una división superior y otra inferior, el ser humano se estructura en siete niveles o planos de manifestación, desde el más denso hasta el más espiritual. Aunque todos los teósofos asumen este carácter septuble, la manera de denominar estos niveles varía según los soportes bibliográficos que se puedan consultar. Siguiendo con la terminología de Blavatsky ${ }^{2}$, si el cuaternario inferior se completa con el cuerpo físico, etérico (fuerza vital que alimenta el cuaternario inferior), astral (reflejo de nuestras emociones) y el kama-manas o mente deseante; la triada más elevada se compone de la mente superior, cuerpo búdico (alma espiritual) y finalmente el cuerpo átmico (monádico) o Espíritu. En cambio, C.W. Leadbeater llega a emplear otra terminología, donde además del cuerpo físico, astral, mental y búdico, estarian también el nirvánico, paranirvánico y mahaparanirvanico. ${ }^{3}$

En relación con este autor, resulta de gran interés la consulta de las siguientes publicaciones: STEBBING, Peter, Conversations about Painting with Rudolf Steiner: Recollections of Five Pioneers of the New Art Impulse, Steiner Books, Great Barrington, Massachusetts, 2008; KUGLER, Walter (ed.), Rudolf Steiner: Blackboard Drawings 1919-1924, Rudolf Steiner Press, New York, 2003.

2 BLAVATSKY, H.P., La clave de la teosofia, Editorial Kier, Buenos Aires, 2007, pp. 87-89.

3 LEADBEATER, C.W. El Hombre Visible e Invisible, Editorial Kier, Buenos Aires, 1999, lámina II.
Este último autor explica y defiende la posibilidad de percibir el aura y las formas de pensamiento de la siguiente manera: "En el ojo humano, tenemos materia sólida y líquida. Supongamos que cada uno de estos estados de materia fuese capaz de recibir impresiones separadamente, pero cada una tan sólo del tipo de materia al cual corresponde. Supongamos también que unos hombres poseyesen la primera de estas formas de visión, y los otros la segunda. Cada una de estas dos clases de hombres tendría indudablemente un concepto tan extraño como imperfecto de nuestro mundo. Imaginemos ahora que dos hombres, uno de cada tipo, se detienen en la orilla del mar; el primero, no pudiendo ver sino la materia sólida, sería completamente inconsciente del océano que se extendería ante él, pero vería la vasta cavidad formada por el lecho del mismo, con todas sus sinuosidades. Los peces y demás habitantes de las profundidades del océano, se le aparecerían flotantes en el aire por encima de este enorme valle." ${ }^{4}$ Cuando el vidente teósofo detecta emanaciones áuricas procedentes de una persona, estas aparecen como vibraciones radiantes y formas flotantes, pudiéndose producir una similitud visual con lo que se observa en los fenómenos hipnagógicos. ${ }^{5}$ Todas estas cuestiones se convertirian en motivo de estudio para algunos pintores modernos. De hecho, en palabras de Juan Ramón Ramírez: "Lo interesante no es que los artistas pudieran llegar a creerse todos los supuestos de la teosofía sino que se sintieran animados por ella para inventarse historias, paisajes o emociones sin recurrir al código naturalista dominante hasta entonces. Esta fue básicamente la operación de Kandinsky, Mondrian, Kupka o Malevich.

La abstracción vino acompañada de un importante corpus teórico, algo que sí pudo haber
LEADBEATER, C.W., El hombre visible e invisible, Editorial Kier, Buenos Aires, 1980, pp. 26-27.

Este término se refiere a un estado mental que se produce entre la vigilia y el sueño, es decir, se origina justo antes de dormir y también en el momento antes de despertarse. Este nivel hipnagógico se ha empleado para estimular todo tipo de visualizaciones, ideas y reflexiones creativas, tal y como lo solía practicar el científico Thomas Edison. Sobre este tema se puede consultar en BELANGER, Michelle, Psychic Dreamwalking: Explorations at the Edge of Self, Newburyport, Massachusetts, Weiser Books, 2006. 
sido estimulado, por la propensión teorirreica de la teosofía." 6

Este campo áurico se complementa tanto con los pensamientos, como la sección más refinada y radiante, y el aspecto del deseo, siendo este último el que más influye en la conformación del aura de un hombre no evolucionado. En palabras de C.W. Leadbeater: "Todos los estudiantes saben que lo que se llama el aura del hombre, es la parte externa de la sustancia etérea de sus cuerpos superiores, de aquella sustancia que los compenetra y trasciende en mucho los límites de su cuerpo físico, el más pequeño de todos. También saben que dos de nuestros cuerpos, el mental y el de deseos, son los que tienen más particularmente que ver con lo que llamamos formas de pensamiento."

Esta observación del aura estaba directamente cohesionada con la visión astral, lo que a su vez llegaría a relacionarse con la exploración de la Cuarta Dimensión, tal y como lo defiende el propio Leadbeater, según lo anota la historiadora del arte Linda D. Henderson. ${ }^{8}$

La apariencia o forma de un pensamiento depende de su tipología, la calidad del mismo y la precisión del pensamiento ejecutado. Para ello, el libro Formas del Pensamiento muestra toda una serie de ejemplos posibles de tales condiciones de manifestación en base a emociones y experiencias vitales. Annie Besant y C. W. Leadbeater plantearon que estas formas de pensamiento eran similares a las figuras de Chladni ${ }^{9}$, formadas por la vibración de una placa de vidrio o latón sobre la que se extiende una fina arena. También, comprobaron que había ciertas similitudes con los diseños intrincados y configurados por un péndulo sobre el cual

$6 \quad$ RAMíREZ, J.A., El objeto y el aura. (Des)orden visual del arte moderno, Akal, Madrid, 2009, p. 177.

7 BESANT, Annie; LEADBEATER, C.W., Formas del Pensamiento, Editorial Humanitas, Barcelona, 1986, p. 19.

8 DALRYMPLE HENDERSON, Linda, The Fourth Dimension and Non-Euclidean Geometry in Modern Art, Princeton University Press, Princeton, New Jersey, 1983, p. 32.

9 Besant y Leadbeater habían leído los trabajos del físico alemán Ernst Chladni (1757-1827), que empleaba las vibraciones acústicas para reproducir manifestaciones tanto del mundo visible como invisible. Los teósofos analizaron el descubrimiento de Chladni como un canal a través del cual contactar con entidades de las esferas superiores. En el libro de ambos autores, se analizan los descubrimientos de este físico, incluyendo el estudio de vibraciones energéticas generadas por pensamientos y emociones. Consultar en BESANT, Annie; LEADBEATER, C.W., Thought-Forms, Theosophical Publishing Company, London, 1901, pp. 16-17. se había añadido un lapicero y las ilustraciones que se proporcionan en el libro Vibration Figures de Frederick Bligh Bond ${ }^{10}$, arqueólogo que empleaba métodos de canalización de los monjes medievales.

Con el objetivo de demostrar que el aura se relaciona también con nuestros pensamientos, Annie Besant y C. W. Leadbeater recopilaron y registraron toda una serie de tipologías ideáticas, que fueron representadas bajo sus indicaciones en distintas ilustraciones. Estas posturas también son defendidas por Alan Peter Garfoot al anotar lo siguiente: "La telepatía incluye el aura humana ya que cuando el sentido del aura humana es desarrollado este puede ser usado para detectar vibraciones en el éter del hiperespacio y emplea el subconsciente para decodificar esas vibraciones y pasarlas al consciente como un mensaje con su única propia impresión a través de la cual la naturaleza del mensaje telepático puede ser entendido." 11

Por otro lado, tal y como lo concreta Rudolf Steiner, se podrían llegar a distinguir los colores de tres tipos de auras. En la primera, están los colores que tienen en mayor o menor grado un carácter de falta de transparencia y opacidad; en segundo lugar están aquellos colores que son prácticamente luz; en el tercer puesto, encontramos colores brillantes y radiantes, que iluminan el espacio que ocupan. ${ }^{12}$ Estos colores se llegan a compenetrar simultáneamente con las formas más variadas, por este motivo, "el aura viene a ser un fenómeno extraordinariamente complicado, pues en cierto modo se trata de tres auras que se encuentran una dentro de la otra y que se compenetran mutuamente." ${ }^{13}$

De la misma manera que cada individuo muestra un aura única respecto al resto, Walter Benjamin derivaría este planteamiento para explicar que cada obra de arte se manifiesta con un tiempo y un espacio determinado desde su creación, asumiendo ésta su unicidad o aura. En una línea similar, encontramos los comen-

10 Para obtener más datos sobre este arqueólogo psíquico, remitirse a NICKELL, Joe, "Dowsing mysterious sites", The Skeptical Inquirer, 27-3, Amherst, New York. 2003, p. 14.

11 GARFOOT, A.P., Dawn of the Neo-Modern: Art, Humanism \& The Meme, Lulu Publishers. Raleigh, North Carolina, 2010, p. 173.

12 Para más información, consultar en STEINER, Rudolf, Teosofía. Introducción al conocimiento suprasensorio del mundo y al designio del hombre, Editorial ECE, Buenos Aires, 2005, pp. 139-141.

13 IBÍDEM, p. 140. 
tarios de Mondrian: "Se trata de saber si podemos ver ese cambio que experimenta la materia. Los ocultistas llegan a suponer que algo de la esfera espiritual del agente queda adherido al objeto. De ser así, la extraña sensación que experimentamos en los museos de arte antiguo podría explicarse por una especie de oposición de la esfera espiritual antigua a la nueva, y viceversa." ${ }^{14}$ En cambio, para Jean-François Lyotard este fenómeno se puede llegar a explicar de otra manera: "El aura es el conjunto de imágenes, que elevándose de la memoria involuntaria, tiende a agruparlas alrededor del objeto, el halo de luz que envuelve un objeto de la naturaleza o del arte con su armonía (sus correspondencias, como afirma Baudelaire; sus asociaciones, dice Freud) y le da la profundidad de un lejano temporal, el Houch del poema de Goethe Ueber alien Gipfeln..... (Más allá de todos los picos de las montañas) - el cuerpo, en resumen, que le genera hablarnos. No hay aura sin continuidad en el tiempo de la psique y de la tradición. Es lo mismo con lo sublime, cuyo sentimiento de aura es quizás sólo un caso particular de él." ${ }^{15}$

\section{Consideraciones cromáticas sobre la temática analizada}

Junto a los trabajos de Leadbeater y Besant, no se debe olvidar el libro Teosofía: una introducción a los procesos espirituales en la vida humana y en el cosmos (1904) de Rudolf Steiner, convirtiéndose en otro de los manuales de consulta por parte de numerosos artistas, entre los que se encontraba especialmente Wassily Kandinsky.

De acuerdo al estudio visionario sobre los cromatismos del aura y las formas de pensamiento de C. W. Leadbeater, debemos subrayar el siguiente comentario: "Para estudiar concienzuda y minuciosamente los diversos cuerpos, debemos familiarizarnos con el significado general de los varios colores que presentan, según se ve en la lámina I. De las combinaciones de los colores primordiales resulta una casi infinita variedad de colores secundarios. Procuré

14 MONDRIAN, Piet, Arte plástico y arte plástico puro, Editorial Víctor Leru, Buenos Aires, 1961, p. 95.

15 LYOTARD, J.F., The Assassination of Experience by Painting, Leuven University Press, Monory, Leuven, 2013, p. 175. presentar lo más aproximadamente posible el exacto color que expresa cada emoción determinada. Sin embargo, las emociones humanas no son casi nunca homogéneas, y el observador debe clasificar constantemente los matices indeterminados y analizar los numerosos factores de su composición.”16

En correspondencia con este párrafo anterior, y en palabras de Juan Antonio Ramírez: "Algunos inventores primitivos de la abstracción, bebieron con avidez variable en las fuentes del esoterismo y de la teosofía. No hace falta sostener la tesis (demasiado extrema) de que esas corrientes del pensamiento condujeron a tales resultados pictóricos, pues ya conocemos la complejidad de los fenómenos artísticos y la multiplicidad de pulsiones presentes en un fenómeno como la abstracción. El caso de Leadbeater ha sido estudiado, sacando a colación, además de sus láminas sobre los colores y las disposiciones del aura, la forma mental de las composiciones musicales." ${ }^{17}$

Para los videntes teósofos, el aura demostraba diferentes valores cromáticos, dependiendo de su conexión con los deseos y el carácter o calidad de los pensamientos, proporcionando en sus libros ejemplos diversos de cómo interpretar los colores del aura en relación con emociones, sentimientos o situaciones que se pudieran dar en la experiencia cotidiana, sirva de ejemplo, como el azul marino es interpretado como el verdadero sentimiento religioso, mientras que el rojo simboliza la ira; el amarillo se conexiona más con el intelecto superior y el negro siempre va a asociado con las intenciones perversas y negativas. Igualmente, para Rudolf Steiner, "muy diferente es el aura según los distintos temperamentos y disposiciones de ánimo de los hombres; diferente también según los grados de desarrollo espiritual." ${ }^{18}$

Esta condición evolutiva en el individuo resulta fundamental en la conformación cromática del aura, tal y como es explicado a continuación: "El color de una cualidad determinada en el cuerpo causal aparecerá también en el mental y en el astral; pero a medida que el ego desciende, el matiz de dicho color será menos delicado, brillante y etéreo. Es tal la diferencia de aspecto de la gama de colores en cada plano, que no se puede dibujarlos con exactitud, y

\footnotetext{
LEADBEATER, 1980, opus cit., p. 85.

RAMÍREZ, opus cit., pp. 176-177.

18 STEINER, opus cit., p. 136.
} 
para dar idea de ello es preciso establecer gradaciones que definan sus características, pues aun la octava inmediata superior a la física, mientras se halla sujeta a las limitaciones del cerebro físico, traspone los límites de cuanto la inteligencia permite imaginar.

Los colores astrales inferiores son sombríos y mates, comparados con los puros tonos de los planos superiores; pero a pesar de su rusticidad, son luminosos; más bien parecen sombríos resplandores de una hoguera, que colores obscuros en el sentido literal de la palabra." ${ }^{19}$ Estas características anteriores son trasladadas a la aplicación cromática que desarrolla Wassily Kandinsky, según el investigador Philippe Sers: "Cada color se identifica primero por su resonancia interna, que es, por su acción sobre el alma. En el texto, esta resonancia interior se define directamente en el párrafo de tres maneras: su función psíquica básica, su génesis en el orden de los colores que se impone en nuestra mente y que se expone como una especie de cromogonía, ontogénesis del círculo de color $y$, finalmente, por su significado espiritual que surge de manera natural los dos anteriores. Los colores se definen también al mismo tiempo por la asonancia: evocando resonancias psíquicas similares a ellos." 20

Algunos de los artistas modernos no sólo leyeron los anteriores tratados, sino que especialmente analizaron las pinturas e ilustraciones, que se insertaron en su interior. En Hombre visible e invisible, las láminas son dibujadas y pintadas del natural por Mauricio Prozor y posteriormente copiadas por Gertrudis Spinck con un procedimiento especial para reproducirlas fielmente en fotograbado. En el caso de Formas del Pensamiento, la elaboración de las ilustraciones a color fue ejecutada por el paisajista John Varley y los teósofos Maurice Prince y Miss Macfarlane, siendo algunas de ellas verdaderas pinturas abstractas. La materialización de estas láminas a raíz de las visiones de Besant y Leadbeater venía marcada por el carácter de la forma de un pensamiento, que dependía tanto del asunto sobre el que se está reflexionando, así como de la calidad del mismo. En este sentido, si los pensamientos de carácter indefinido se manifiestan como una especie de nebulosa, los que están bien determinados conllevaban

LEADBEATER, 1980, opus cit., p. 76.

20 SERS, Philippe, Kandinsky. Philosophie de l'abstraction: L'image métaphysique, Skira, Genève, 1995, p. 67. composiciones más nítidas y sólidas. A partir de aquí, ambos investigadores empezaron a documentar toda una dilatada tipología: lo lujurioso o maligno, mediante tentáculos verdes fangosos; la ira descontrolada con destellos rojos y naranjas; la devoción mediante un cono azul. Igualmente, analizaron la traslación cromática de sentimientos positivos, como la afección pura, el amor intenso, la paz; mientras otros se enmarcaban dentro de pautas negativas, caso del egoísmo, la ambición, el odio y la ira, entre otros. Dispusieron de la traducción cromática de situaciones tan diversas, como un accidente en la calle, un entierro, el encuentro con un amigo, la contemplación de un cuadro, e incluso la música de Mendelssohn, Gounod y Wagner.

\section{Las propuestas abstractas de los artistas vanguardistas}

Entre los numerosos creadores que se han acercado a la literatura teosófica, con el objetivo de extraer distintos parámetros teóri$\cos ^{21}$, para posteriormente ser volcados en la producción pictórica, bajo premisas subjetivadas, encontramos primordialmente a Wassily Kandinsky, quizás uno de los pintores que más alusiones ha realizado en el contexto de las Vanguardias Históricas en conexión con la representación abstracta de las auras ${ }^{22}$ y las formas de pensamiento. De hecho, tal y como defienden numerosos autores, como Annegret Hoberg y Helmut Friedel, en referencia a este pintor ruso: "Conocía los trabajos de Helena P. Blavatsky, Edouard Schuré y Annie Besant, así como el libro clásico de C.W. Leadbeater Formas de pensamiento. Se ha probado que tenía conocimiento de las lecturas de Rudolf Steiner y que estuvo en contacto con el círculo de Karl

21 Resulta relevante el caso de la pintora sueca Hilma af Klint en relación con los numerosos trabajos geométrico-abstractos que realizó a principios del siglo XX. Para obtener más información al respecto, se debe consultar SARRIUGARTE, I. (2019): “Mediumnismo y arte. El caso de Hilma af Klint: de la mano dirigida a la mano intuitiva", La Colmena-Universidad Autónoma del Estado de México, 102: 85-103.

22 Debemos recordar que este artista realiza diversas anotaciones en los márgenes del libro titulado Jenseitiger Mensch (1925) de Emil Mattiesen, donde se abordan conceptos como el aura, el mediumnismo y la telepatía. 
Wolfskehl y Ludwig Klages ${ }^{23}$ en Munich, y con el neurólogo Albert von Schrenk-Notzing, que también se había centrado en las percepciones extrasensibles y el fenómeno de la parapsicología. En los libros ilustrados por estos autores Kandinsky encontró la inspiración para las formas que podrían adaptarse a su comportamiento para expresar lo inmaterial." ${ }^{24}$

Igualmente, para Sixten Ringbom ${ }^{25}$, el pintor estuvo muy interesado en cuestiones paranormales, místicas y espirituales, especialmente en algunas formas de meditación y visualización. En la misma línea, se expresa Hajo Düchting: "Se dedicó al estudio de las ciencias ocultas, interesándose por los escritos de Rudolf Steiner y Helena Petrovna Blavatsky, fundadora de la Sociedad Teosófica." ${ }^{26}$

Además, debemos anotar su interés por la fotografía del pensamiento, es decir, la posibilidad de captar mediante placas sensibles algo que es tan intangible e invisible como los pensamientos y darle una proyección visible ante nuestros ojos. Su predilección hacia estos campos de la investigación se pudo observar durante toda su vida, pero especialmente tomaron una fuerte consistencia entre los años 1904-1920, periodo donde se estaban produciendo numerosas exploraciones psíquicas a nivel internacional. Son años donde diferentes investigadores, mediante el uso de métodos científicos, intentaron demostrar la existencia de un entramado espiritual. Por ejemplo, debemos anotar los esfuerzos llevados a cabo durante 1920 por Arthur Conan Doyle, cuando intentó captar las famosas hadas de Cottingley,

23 Sobre las actividades culturales que realizan tanto el poeta Karl Wolfskehl como el pensador Ludwig Klages, se puede consultar en WASTON LONG, R.C., "Kandinsky's Vision of Utopia as a Garden of Love", Art Journal, 43-1, London, 1983, p. 56. Especialmente sobre este último, resulta destacable la siguiente publicación: LEBOVIC, Nitzan, The Philosophy of Life and Death: Ludwig Klages and the Rise of a Nazi Biopolitics, New York, Palgrave Macmillan, 2013.

24 HOBERG, Annegret; FRIEDEL, Helmut, Kandinsky. Absolute Abstract, Prestel, London, 2008, p. 80.

25 Para obtener más datos, dirigirse a RINGBOM, Sixten, "Art in the Epoch of the Great Spiritual, Occult Elements in the Early Theory of Abstract Painting", Journal of the Warburg and Courtauld Institutes, XXIX, London, 1996, p. 400. También, se presenta de gran valor documental la consulta del mismo autor The Sounding Cosmos: A Study in the Spiritualism of Kandinsky and the Genesis of Abstract Painting, Åbo Akademi, Åbo, Finland, 1970.

26 DÜCHTING, Hajo, Kandinsky 1866-1944. Una revolución pictórica, Taschen, Köln, 2012, p. 26. trabajo que lo único que consiguió fue reforzar la actitud escéptica de la comunidad científica hacia este tipo de conjeturas, lo que no impidió, por otra parte, que Kandinsky se siguiera acercando a estas investigaciones paranormales. En cualquier caso, sin entrar en la consistencia científica de dichos experimentos, para el artista ruso su interés por la fotografía del pensamiento se conexionaba directamente con la apertura y el advenimiento de una nueva era en la evolución de la conciencia humana, que lo denominaría la Gran Época Espiritual: "Al final quisiera añadir, que según mi opinión, nos acercamos cada vez más a la época de la composición consciente y racional......., que ya estamos en el tiempo de la creación útil y, finalmente, que este espíritu de la pintura está en relación orgánica directa con la ya iniciada construcción del nuevo reino espiritual, ya que este espíritu es el alma de la época de la gran espiritualidad." ${ }^{27}$

Enfrascado dentro de la moda por explicar lo invisible, Kandinsky pretendió aportar su granito de arena a un tema, que nunca dejaba de ser resbaladizo y a la vez atrayente. En este ámbito, se sintió muy interesado por los estudios teosóficos, que trataban de representar cromáticamente el aura y las diversas formas del pensamiento y para ello hizo uso de los libros de Leadbeater, Besant y Steiner, conociendo a este último cuando acude a sus conferencias en Munich y Berlín. ${ }^{28}$ De acuerdo a Hajo Düchting: "Parece que Kandinsky se sintió estimulado a representar las formas luminosas con aura gracias a la lectura del clásico libro teosófico Formas del pensamiento, escrito por Annie Besant y C. W. Leadbeater, publicado en alemán en 1908. Pretendía poner de manifiesto lo espiritual mediante formas y colores abstractos. Intentó confirmar la secreta relación interna entre el estímulo del color y la impresión espiritual y psíquica sobre el espectador, apoyándose en las numerosas especulaciones sobre las conexiones sinestésicas, habituales sobre todo en los círculos simbolistas. Las ideas teosófico-místicas de las vibraciones del alma y las delicadas oscilaciones tuvieron que signi-

\footnotetext{
27 KANDINSKY, Wassily, De lo espiritual en el arte, Editorial Labor, Barcelona, 1992, pp. 121-122.

28 SHEPPARD, Richard, Modernism - Dada - Postmodernism, Northwestern University Press, Evanston, Illinois, 2000, p. 409.
} 
ficar para él, más que atrevidas construcciones doctrinarias, una auténtica prueba de fe." ${ }^{29}$

También recopiló información mediante la lectura de la revista teosófica de Steiner conocida como Luzifer-Gnosis. De hecho, en los apuntes del pintor ruso se transcriben varios pasajes de una serie de artículos de Steiner, entre otros, el titulado Von der Aura des Menschen (El Aura del Hombre). ${ }^{30}$

El interés cromático de Kandinsky es descrito por Michel Henry de la siguiente manera: "Conocemos, es cierto, la pasión de Kandinsky por el color, pasión que suscitó su vocación de pintor y marcó su obra desde el principio, especialmente todo el período fauve anterior al descubrimiento de la abstracción. Sabemos también que fue esa pasión por los colores lo que empujó a Kandinsky a elaborar una teoría sistemática del color. Ésta se impone a nosotros con amplitud y precisión inigualadas. Su tesis maestra es precisamente que cada color tiene una tonalidad afectiva propia. En esta medida, el color oculta su ser a la luz de lo visible." ${ }^{31}$

Podemos observar en este párrafo anterior que los colores son coaligados a diferentes tipos de emociones, tal y como lo había propuesto previamente el propio Leadbeater. Dicha conexión es explicada nuevamente por parte de Michel Henry de la siguiente manera: "Cierto es que hay una apariencia de color que se extiende sobre el objeto, confundiéndose, al parecer, con su superficie, con su extensión. Pero ese color percibido sobre el objeto o, análogamente, sobre el cuadro - ese color objetivo, noemático, como dice Husserl- es solamente la proyección en el objeto de una sensación de color que no es más que en tanto es sentida, y que no es sentida más que en tanto ella se siente a sí misma: en lo invisible de su afectividad." ${ }^{32}$

Bajo el prisma estético de Kandinsky, se quiere alcanzar un acercamiento a la verdadera realidad espiritual, y para ello se debe superar la percepción física. Ante la oferta materialista de la civilización contemporánea, el pintor ruso apostó directamente tanto en creación pictórica como en producción literaria por un enfoque espiritual, que se vería concretizado en su manifiesto Über das Geistige in der Kunst

29 DÜCHTING, opus cit., p. 38.

30 Este texto se encuentra publicado en Lucifer-Gnosis, Jan-Apr., 34, Berlin, 1904, pp. 110-137.

31 HENRY, Michel, Ver lo invisible. Acerca de Kandinsky, Siruela, Madrid, 2008, p. 88.

32 IBÍDEM
(1912). Por este motivo, en opinión de Marc J. LaFountain, este artista: "Buscó violar las apariencias diarias del mundo en el nombre de una comprensión más clara, simple y pura de la naturaleza y el sentido de las cosas, así también lo hizo Husserl. El trabajo de Husserl, mientras que era sospechoso de tendencias materialistas dominantes del conocimiento, estaba, por supuesto, desprovisto de las características noumenales y ocultistas que pintaba el pensamiento y los cuadros de Kandinsky. La Crisis de las Ciencias Europeas y la Fenomenología Trascendental, por ejemplo, fue, como el trabajo de Kandinsky, escrito contra una serie de agitación y perdida interior, contra el mismo legado de la óptica racional y el objetivismo que concierne a Worringer. Mientras las tendencias transcendentales de Kandinsky y Husserl fueron nostálgicas y utópicas (por ejemplo, las leyes del Espíritu y del cosmos de Kandinsky y las esencias irreales perfectas de Husserl), lo que distingue a Husserl de Kandinsky es la eliminación por parte de Husserl del aura esotérico y místico de las esencias al situar los interrogantes en el significado dentro de la practibilidad (y seguridad) de la epistemología." ${ }^{33}$

Si el punto de partida del pintor fueron las teorias teosóficas de Besant, Leadbeater y Steiner, donde se explicaba cromáticamente las diferentes tipologías áuricas y formas del pensamiento, mediante una materialización cromática, es muy probable que Kandinsky únicamente hiciera uso de las ideas generales para luego posteriormente construir sus propias teorías hipotéticas, sin olvidarnos de su interés por el estudio sinestésico, donde se aplica la sustitución o coincidencia de un sentido con otro, como en el fenómeno de los colores auditivos o sonidos visibles ${ }^{34}$. Debemos tener en cuenta que tanto para los estudiantes de las teorías de Rudolf Steiner, como para Kandinsky, la sinestesia era entendida como una señal de avance en el camino espiritual. ${ }^{35}$ No sólo Kan-

33 LAFOUNTAIN, Marc J., Dali and Postmodernism: This Is Not an Essence, University of New York Press, New York, 1997, pp. 11-12.

34 Para más información, consultar en DE CORDOBA, M. J. et al. (2014): Sinestesia. Los fundamentos teóricos, artísticos y científicos, Granada, Ediciones Fundación Internacional Artecittà.

35 LISTENGARTEN, Julia, "Michael Chekhov and the visual arts: influences, synergies, collaborations", en AUTANT-MATHIE, M.C.; MEERZON, Yana (eds.), The Routledge Companion to Michael Chekhov, Routledge, New York, 2015, p. 257. 
dinsky estaba familiarizado con esta facultad, ya anteriormente se había convertido en uno de los temas estrella para los simbolistas. Incluso desde el ámbito de la literatura se habían desarrollado propuestas, como la realizada por Arthur Rimbaud en su poema titulado Vocales, donde asigna colores a los sonidos fonéticos de las vocales: negro-A, blanco-E, rojo-I, azul-0, verde-U.

Todos estos planteamientos teóricos e hipótesis transcendentales abrieron numerosas posibilidades intuitivas y creativas para los artistas encaminados a desarrollar las pautas del lenguaje abstracto. Ciertamente, la apuesta por la abstracción asumía una correlación paralela con la visión más espiritual y con todos sus planteamientos más novedosos. Bajo este prisma personal de inducir cambios en la mentalidad y la conciencia occidental, Kandinsky encamina su producción con el objetivo de formular un nuevo escenario, abriendo de este modo un amplio abanico de posibilidades y recursos para la pintura, camino que no sólo sería imprimido por Kandinsky, sino por la mayoría de los neoplasticistas, suprematistas y otras tendencias derivadas de estas primeras.

Entre los principales trabajos de Kandinsky cohesionados con las formas del pensamiento y el aura, encontramos Varios círculos (1926), de hecho, estos mismos son empleados por Leadbeater en las láminas XI, XII (impulso súbito de devoción) y la número XIII (un acceso de cólera). En esta última imagen, podemos observar formatos circulares y espirales en zig-zag, que también se aprecian en otras propuestas de Giacomo Balla, caso de Lineas de velocidad (1913) y el estudio preliminar en témpera para Spessori d'atmosfera (1913). De igual modo, los diseños en zig-zag se emplean en Temporal patriótico (1924) de Fortunato Depero ${ }^{36}$. También, estas secciones curvilíneas de color negro se asemejan a las formas serpentinas de Amarillo, rojo y azul (1925) de Kandinsky.

En Estudio del color con cuadrados (1913), Composición VIII (1923), Círculos en un círculo (1923) y Acento en Rosa (1926) se observan

36 Los futuristas Boccioni, Balla y Russolo estaban convencidos de que los rayos $\mathrm{X}$ podían ayudar a registrar los aspectos invisibles de la realidad, caso de las señales residuales del movimiento de los cuerpos y las emanaciones luminosas producidas por la cabeza y proyectadas en el aura. Remitirse a CELANT, Germano, "Futurismo Esoterico", II Verri, 33-34, Milano, 1970, pp. 108-117. formas circulares con sus respectivas auras. De hecho: "El círculo ha sido siempre interpretado como símbolo de perfección. En esta obra Kandinsky potencia este simbolismo con la solemnidad del fondo oscuro, dando al lienzo un matiz místico a la vez que cósmico, como si estuviera representando un orden superior. Tal composición dista mucho de la frialdad de su etapa anterior en Weimar." ${ }^{37}$

Los investigadores alemanes Annegret Hoberg y Helmut Friedel apuntan los siguientes datos: "En una carta a un amigo, al historiador del arte alemán Will Grohmann, Kandinsky describe el círculo como (1) la forma más modesta, pero se reivindica a si mismo de manera incondicional, (2) precisa pero variable e inagotable, (3) simultáneamente estable e inestable, (4) simultáneamente ruidosa y sumisa, (5) una simple tensión que conlleva tensiones incontables dentro de éste... El círculo ciertamente poseía poderes evocativos de un orden celestial, pero Kandinsky lo usaba como un medio formal, una forma perfecta capaz de estructuras y posibilidades espaciales infinitas, cruzándose planos de tonalidades brillantes y colores transparentes. Reconociendo su fuerza diná-

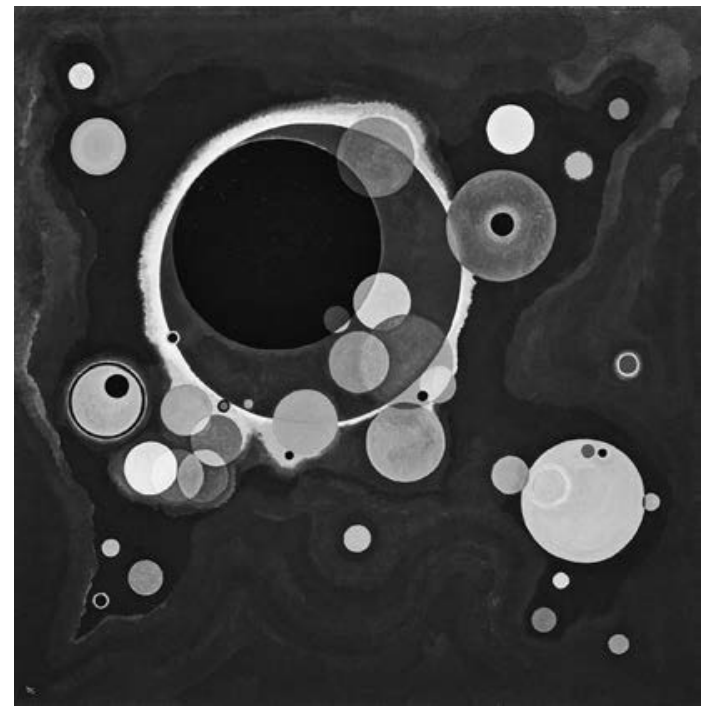

Fig. 1. Wassily Kandinsky, Varios círculos, óleo sobre lienzo, 1926 Fuente: The Solomon R. Guggenheim Museum, New York.

mica que le inspiró continuamente, el artista usará el círculo de nuevo en sus pinturas de

\footnotetext{
${ }_{37}$ TRIADÓ, Xavier, Kandinsky, Tikal Ediciones, Madrid,
} 2009, p. 176. 


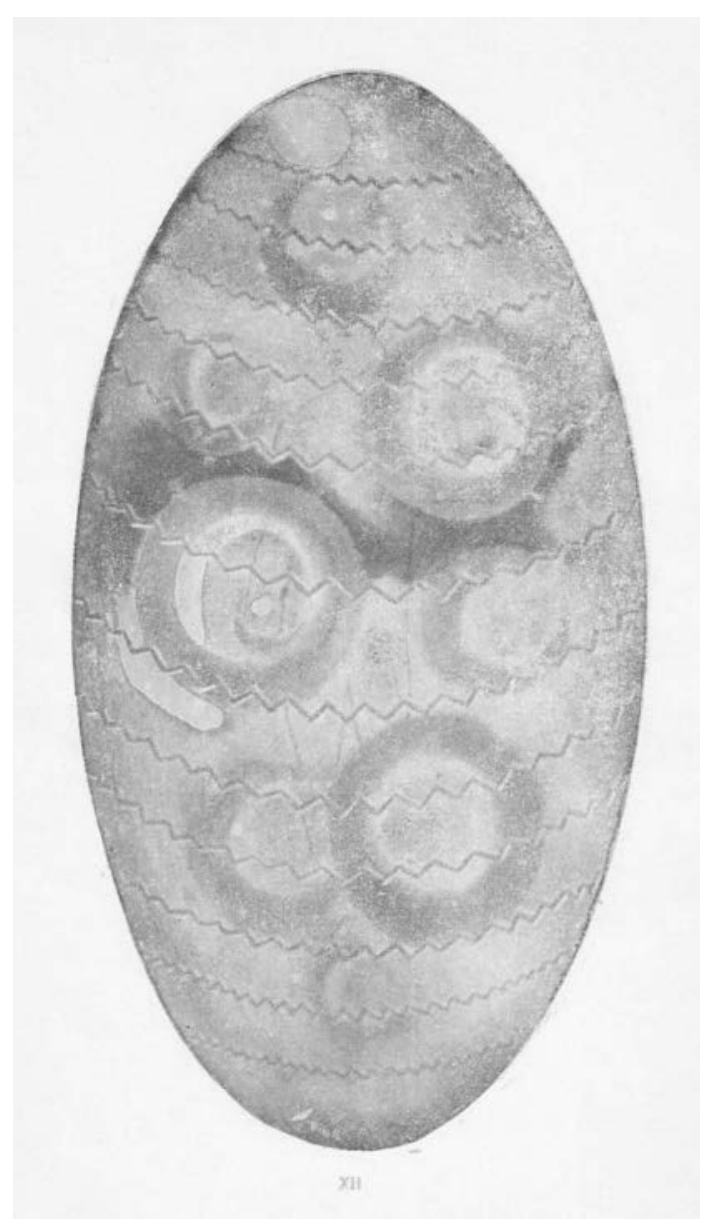

Fig. 2. Lámina $\mathrm{n}^{\circ}$ 12: Un impulso súbito de devoción. Fuente: BESANT, Annie; LEADBEATER, C. W. Thought-Forms. London: Theosophical Publishing Company, 1901.

finales de los años 20 y en los años 30." ${ }^{38}$ Por otro lado, según Hans Konrad Roethel: "Debido a que el círculo poseía para él la indicación más clara de la cuarta dimensión, él la prefería como un elemento ante otras formas geométricas como el triángulo o el cuadrado. De este modo al usar el círculo, movimiento - como la verdadera esencia del tiempo - se vuelve un componente visual de sus pinturas.... Como un contrapunto musical, no hay un tema, formalmente hablando, para el cual las otras formas están subordinadas, pero todos los elementos tienen su propia vida independiente, y esto es por su constelación que hace que se vuelvan una evidencia de resultado tanto el movimiento como la emoción." 39

HOBERG; FRIEDEL, opus cit., p. 150.

39 ROETHEL, H.K.; BENJAMIN, J.K., Kandinsky, Phaidon, Oxford, New York, 1979, p. 136.
En el cuadro Amarillo, rojo y azul (1925), el enfrentamiento sintomático entre las dos fuerzas que asumen continencias diferenciadas tanto formales como cromáticas refleja un visible acercamiento a la simbología de Leadbeater: $\mathrm{Si}$ por un lado predomina el amarillo marcado por una intelectualidad superior, por otro, se observa una avalancha de formas caóticas, mediante colores azules fuertemente oscurecidos, rojizos y negros, característicos de la malicia, el sentimiento religioso egoísta y la cólera. ${ }^{40} \mathrm{En}$ esta obra, se aprecian trazos radiantes desde el punto focal del rectángulo superior, líneas que recuerdan a las empleadas en la lámina número 26, donde se muestra el cuerpo causal de un adepto. Para Rudolf Steiner, "todo lo que es indicio de actividad espiritual tiene más bien la figura de rayos que se despliegan desde adentro, mientras que todo lo que proviene de la vida animal tiene la forma de nubes irregulares que fluyen a través del aura." ${ }^{41}$ Estas características asimismo se pueden apreciar en Improvisación 27 (jardín del amor II) (1912).

En relación con Composición V(1911), Alain Besançon realiza el siguiente comentario: "El título alude a la música. Las formas luminosas rodeadas de un aura deben algo al clásico de la teosofía Las formas del pensamiento, de Annie Besant. Se ha cruzado el umbral. La pintura abstracta ha sido concebida, pensada como tal, fundada." ${ }^{42}$ Por otro lado, en la obra Cuadro con borde blanco (1915), se puede apreciar la imagen de San Jorge representada mediante un aura de color azul, no resultando este hecho una coincidencia, ya que como bien comenta Hajo Düchting, "tanto Marc como Kandinsky consideraban el azul como el color típicamente celestial, es decir, espiritual." ${ }^{\text {3 }}$ Si observamos la gama cromática expuesta por Leadbeater en El hombre visible e invisible, comprobamos que la mancha azul de San Jorge tiene profundas similitudes con el color azul que simboliza el sentimiento religioso puro $^{44}$. Esta tonalidad azul se vuelve a reproducir en Pintura azul (1924) y En azul (1925), entre otras tantas. Igualmente, Rudolf Steiner en alusión a este mismo color anota que "de la misma manera se presenta

\footnotetext{
40 LEADBEATER, opus cit., p. 16.

${ }^{41}$ STEINER, opus cit., p 138.

42 BESANÇON, Alain, La imagen prohibida: una historia intelectual de la iconoclasia, Siruela, Madrid, 2003, p. 407.

43 DÜCHTING, opus cit., p. 42.

${ }_{44}$ LEADBEATER, 1980, opus cit., p. 16.
} 
también la de muchas personas religiosas, devotas. Las almas piadosas y las que gustosamente se entregan a una existencia plena de caridad tienen un aura similar." ${ }^{45}$

De igual modo, las formas ovaladas de los dibujos que aparecen en el libro de Leadbeater se vuelven a ver con claridad en la obra Ovalo Espejo (1911). Para finalizar con este pintor, no podemos olvidar Concentración fría (1930), donde se repiten estratos nebulosos de diferentes cromatismos, acompañados de un triángulo y un rectángulo. Estas nubes poco definidas como el triángulo se aprecian de similar manera en las láminas de Formas del Pensamiento, especialmente en la número 53, en alusión a pensamientos de auxilio.

El año 1912 resulta de gran valor documental, ya que van Doesburg comienza a desplegar un notorio esfuerzo literario al convertirse en colaborador del semanal Eenheid, una publicación enfocada a nutrir el interés esotérico de un variado conjunto de lectores que comprendían desde masones, teósofos y rosacrucianos hasta militantes del vegetarianismo, entre otras tipologías. Por ejemplo, sobresalen los extensos textos redactados bajo el epígrafe Especímenes del nuevo criticismo artístico, donde se abogaba por una audaz defensa de los valores superiores tanto en la comprensión como en la manifestación artística, haciendo especial hincapié en la profundización de aquellos estratos versados en las constantes más internas y elevadas.

Este artista, que ya se encontraba interesado desde hace años por la manifestación de las formas áuricas, quedó visiblemente impresionado en 1915, cuando acompañó al artista Erich Wichmann para visitar al pintor Janus de Winter, cuyos trabajos se producían mediante estados de trance y semi-inconsciencia, tanto en la representación de las formas abstractas como de sus paisajes imaginarios y animales extraños. Para Carol Blotkamp: "Winter expresa emociones, observando en los seres los estados mentales que los rodean y no con los ojos, sino centrando su atención psíquica en alguien. Él se basa más o menos en la teoría teosófica de que todo ser humano está rodeado de un aura con colores y formas características." ${ }^{46}$

45 STEINER, opus cit., p. 137.

46 LEMOINE, Serge, Theo van Doesburg. Peinture, architecture, theorie, Philippe Sers Editeur, Paris, 1990, p. 17.
Durante estos años la pintura de van Doesburg está muy influenciada por las propuestas de Janus de Winter ${ }^{47}$. Cuando van Doesburg visita en Laren a Piet Mondrian, durante 1916, seguía con su objetivo de promocionar la obra de Winter. También, en esta misma localidad, tendrá la oportunidad de conocer al compositor Jakob van Domselaer, al matemático y teósofo Schoenmaekers y al pintor Peter Alma. En una carta fechada el 7 de febrero, van Doesburg describe este encuentro: "Yo había llevado unos cuantos trabajos de Winter. Ellos encontraron el trabajo muy bello pero no muy espiritual. Tenían la impresión de una transformación de la naturaleza. El D. Schoenmaekers pensaba que eran simples representaciones de visiones astrales. En general me dio la impresión de que van Domselaer y Mondrian estaban totalmente bajo el encanto de las ideas del D. Schoenmaekers." ${ }^{48}$ A pesar de estas impresiones negativas, van Doesburg siguió sin comulgar con la actitud de Mondrian y Schoemaekers respecto a las pinturas de Janus de Winter, y continuó mostrando una visible admiración por su producción.

Entre sus obras más interesantes, destacamos Composición (1915), en clara relación con la ilustración número XXII (el cuerpo mental del hombre evolucionado), donde se remarca en la zona superior del cuerpo, a la altura de la cabeza, una fuerte propensión amarilla, al igual que en su pintura. Pero, especialmente destacable resulta Aura Cósmica (1915), "donde hace clara referencia a sus contactos con la teosofía y con el mundo de De Winter." ${ }^{49}$ Asimismo, este trabajo se podría correlacionar con las láminas número 42 y 54 del libro Formas del Pensamiento, que representan el esfuerzo dirigido a imaginar el Logos penetrando en la naturaleza y pensamientos de auxilio respectivamente.

También, autores como Henry Geldzahler ${ }^{50}$ comentan la práctica habitual de František Kupka y otros compañeros orfistas en la repre-

47 BLOTKAMP, Carel, Mondrian: The Art of Destruction, Reaktion Books Ltd., London, 1994, p. 95.

48 BLOTKAMP, Carel et al. De Stijl: The Formative Years. 1917-1922, The Mit Press, Cambridge, Massachusetts, 1982, pp. 9-10.

49 CREGO Charo, El espejo del orden. El arte y la estética del grupo holandés De Stijl, Akal, Madrid, 1997, p. 41.

50 GELDZAHLER, Henry, American Painting in the Twentieth Century, The Metropolitan Museum of Art, New York, 1965, p. 72. 


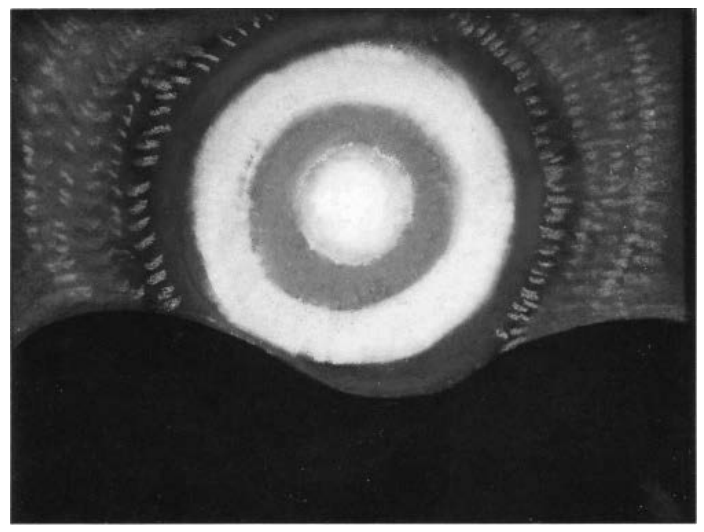

Fig. 3. Theo Van Doesburg, Aura cósmica, pastel sobre papel, 1915. Fuente: Colección particular, Amsterdam.

sentación del sol y su aura, como emanación y fuente de color. Para los investigadores Frank Büttner y Andrea Gottdang ${ }^{51}$, a principios de siglo XX se hicieron más familiares y cercanas diversas nociones esotéricas sobre energías psíquicas que se propagaban a modo de radiación, siendo uno de sus motivos el aura de los individuos, lo que indujeron a artistas como Frantisek Kupka a la búsqueda de nuevas opciones de visualización. No obstante, debemos recordar que este pintor había mostrado dotes y capacidades mediumnísticas desde niño. Esta misma línea argumental se puede encontrar en los textos de Virginia Spate ${ }^{52}$, defendiendo que tanto el trabajo de Kupka como el de los orfistas mantenían la creencia de que el aura era una manifestación lumínica de un nivel superior. Una de estas similitudes formales y cromáticas se encuentra en la lámina número 31 de Formas del Pensamiento, donde se puede observar el estado mental de un actor a la espera del estreno, y en una serie de cuadros de Kupka, que se componen de líneas verticales, caso de Mme Kupka entre Verticales (1911), La Catedral (1912-13) y Reminiscencia de una Catedral (1920-23), entre otras. También, resulta relevante el cuadro El Primer Paso (1910) con toda una serie de círculos, que se aproximan a las obras de Kandinsky.

51 Consultar en BÜTTNER, Frank; GOTTDANG, Andrea, Einführung in die Malerei: Gattungen, Techniken, Geschichte, C.H. Beck, München, 2012.

52 SPATE, Virginia, Orphism: the evolution of non-figurative painting in Paris, 1910-1914, Clarendon Press, New York, 1979, p. 28.

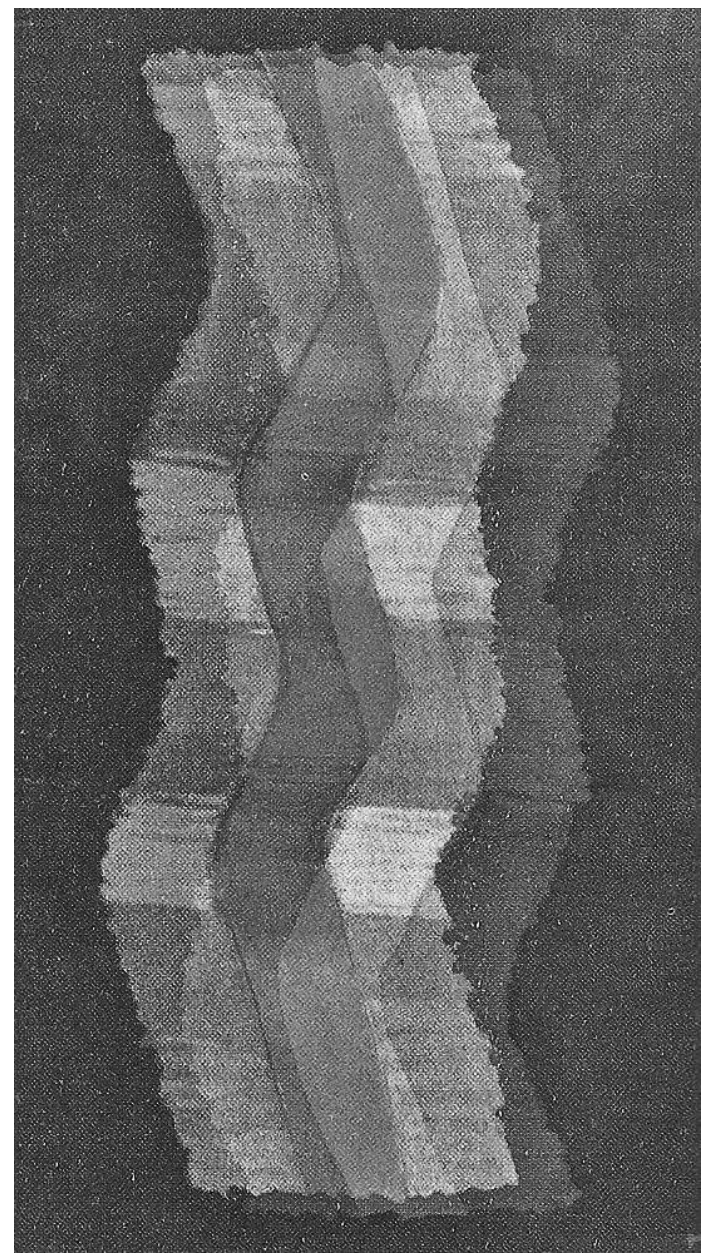

111

Fig. 4. Lámina $\mathrm{n}^{\circ}$ 31. Fuente: BESANT, Annie; LEADBEATER, C. W. Thought-Forms. London: Theosophical Publishing Company, 1901.

\section{Conclusiones}

Desde los postulados teóricos planteados por H.P. Blavatsky en la Doctrina Secreta y los trabajos de C.W. Leadbeater y Annie Besant en Formas del Pensamiento y El hombre visible e invisible, así como Teosofía: una introducción a los procesos espirituales en la vida humana y en el cosmos de Rudolf Steiner, se gestan toda una serie de conjeturas hipotéticas sobre el concepto de aura y las formas de los pensamientos, que serán analizados por diversos artistas ubicados en las tramas renovadoras de las Vanguardias Históricas, convirtiéndose en una plataforma que les permitirá ahondar en campos inéditos de experimentación hasta el momento. Estas estrenadas fuentes de conocimiento e información supondrán no sólo la posibilidad de nuevos retos en el ámbito de la abstracción, sino la justificación de una temá- 
tica que se pretendía sustentar en raíces mucho más espirituales que físico-materiales.

Tanto el aura como las formas de los pensamientos conllevaban una tipología en conexión con la calidad de las mismas y la precisión del pensamiento emitido, tal y como se explica teórica y visualmente en las publicaciones de Annie Besant y C. W. Leadbeater. No todos los artistas se identificaron de una manera creíble con estas suposiciones, pero a una serie de ellos les sirvió como trampolín para recrear nuevas averiguaciones cromáticas, lo que por primera vez permitiría que la abstracción fuera arropada por un extenso corpus teórico-espiritual. Para articular y asegurar este objetivo, artistas como Theo van Doesburg, František Kupka, los futuristas italianos y especialmente el pintor ruso Wassily Kandinsky, tuvieron que sumergirse en estas aportaciones teóricas de orientación teosófica, realizando no sólo consultas sino una decidida reflexión sobre su posterior uso artístico. Tanto desde este apartado especulativo-filosófico como desde la mera experimentación cromática, muchos creadores de la modernidad encontraron un entramado facilitador para su trabajo innovador, que ahondaba en la correlación entre el color y el valor psíquico-emocional. De este modo, dichos pintores posibilitaron el acercamiento a una nueva realidad de carácter intangible, que podía ser analizada e investigada, lo que obligaba a asumir la superación de la barrera físico-perceptiva.

Si en las aportaciones pictóricas de Wassily Kandinsky se sugieren composiciones elaboradas en base a círculos, que asumen ciertas similitudes con algunas de las láminas reproducidas en las obras de Besant y Leadbeater,

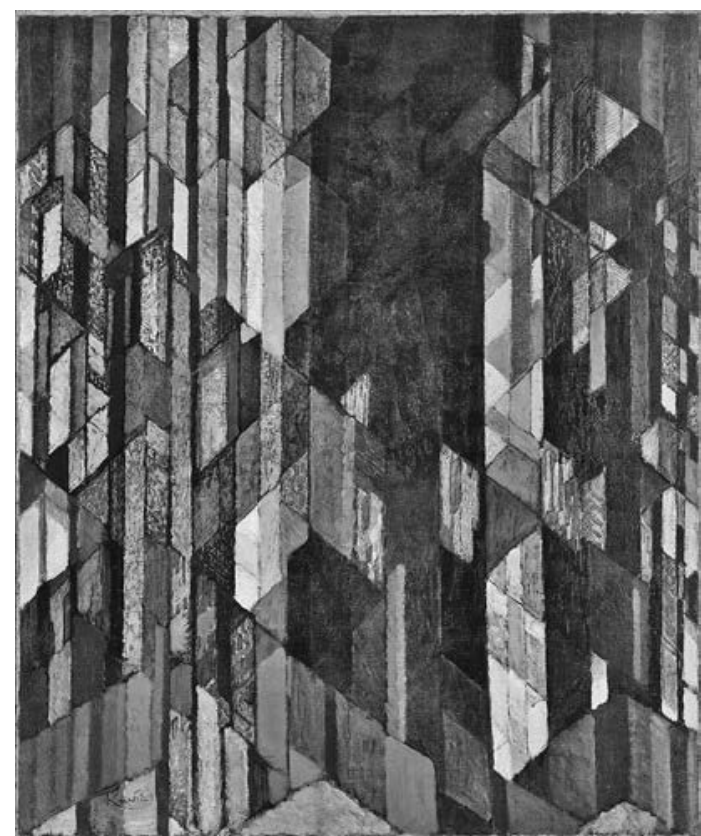

Fig. 5. František Kupka, La catedral, óleo sobre lienzo. 1912-13. Fuente: Museum Kampa, Praga.

igualmente se observan posibilidades comparativas entre dichas láminas y las espirales en zig-zag de Giacomo Balla y Fortunato Depero. En el caso de Theo van Doesburg se produce un acercamiento a los trabajos del pintor Janus de Winter, cuyas propuestas se alineaban con estados de trance, que le permitían extraer las características áuricas que rodeaban a los seres humanos. Igualmente, resultan destacables las representaciones de los orfistas en esta línea, sobresaliendo František Kupka, quien se embarcó en el estudio de la emanación de las energías psíquicas y del aura. 